\title{
ИЗГИБАЕМЫЕ ЭЛЕМЕНТЫ ИЗ СЛАНЦЕЗОЛЬНОГО ЯЧЕИСТОГО БЕТОНА С НАПРЯЖЕННОЙ АРМАТУРОЙ
}

\section{А. МоРозов}

\section{1. Введение}

В настоящее время все большее применение в строительстве находят армирован ные конструкции из ячеистых бетонов. Однако таким бетонам свойственна большая деформативность. При применении ячеистых бетонов в изгибаемых элементах для уменьшения прогибов последних наиболее эффективным мероприятием могло бы быть обеспечение их работы в стадии I, т. е. при отсутствии трещин.

В армированных конструкциях из сланцезольного ячеистого бетона автоклавного твердения арматура получает начальные напряжения около $1000 \mathrm{\kappa} \Gamma / \mathrm{cm}^{2}$ из-за наличия разности значений коэффициентов линейного расширения ячеистого бетона и металла, но это не обеспечивает работу, конструкций в стадии I, так как значительная часть напряжений теряется в течение первых месяцев.

Поэтому в Институте строительства и строительных материалов в 1958-1961 гг. была проведена работа по проверке возможности применения напряженной стержне вой арматуры в изгибаемых элементах из ячеистого бетона. Ввиду отсутствия по этому вопросу данных в литературе, работа была начата с выяснения принципиальной возможности такого применения стержневой арматуры. В первых же опытах, проведенных на изгибаемых элементах из пеносиликальцита, было выяснено, что в этом случае особое значение приобретают вопросы анкеровки арматуры и ее антикоррозионного покрытия,

\section{2. Описание опытов}

В табл. 1 приведены данные об испытанных изгибаемых элементах из сланцезольного ячеистого бетона с напряженной арматурой.

Таблица 1

\begin{tabular}{|c|c|c|c|c|c|c|c|c|c|c|c|c|}
\hline \multirow{2}{*}{ № } & \multirow{2}{*}{$\begin{array}{c}\text { Aрма- } \\
\text { тура } \\
F_{\text {н }}, \\
C M^{2}\end{array}$} & \multirow{2}{*}{$\begin{array}{c}\text { Кол-во } \\
\text { диаметра } \\
\text { арма- } \\
\text { туры }\end{array}$} & \multicolumn{3}{|c|}{$\begin{array}{c}\text { Размеры сечения, } \\
\text { см }\end{array}$} & \multirow{2}{*}{$\begin{array}{l}- \\
- \\
5 \\
\vdots \\
0 \\
0\end{array}$} & \multicolumn{2}{|c|}{$\begin{array}{l}\text { Объемный } \\
\text { вес, } \kappa \Gamma / \mu^{3}\end{array}$} & \multicolumn{2}{|c|}{$\begin{array}{c}\text { Прочность, } \\
\kappa \Gamma / \mathrm{cm}^{2}\end{array}$} & \multirow{2}{*}{$\begin{array}{c}\text { Модуль } \\
\text { упру- } \\
\text { гости } E_{0} \text {, } \\
\kappa \Gamma / c \mu^{2}\end{array}$} & \multirow{2}{*}{$\begin{array}{l}\frac{\pi}{5} \\
\frac{5}{5} \\
\frac{5}{2} \\
0\end{array}$} \\
\hline & & & $h$ & $h$ & $b$ & & $\gamma_{\text {cyx }}$ & $\gamma_{B \pi}$ & 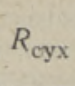 & & & \\
\hline 4 & $\begin{array}{l}1,5 \\
2,3\end{array}$ & $\begin{array}{l}2 \varnothing 10 \\
3 \varnothing 10\end{array}$ & 24 & & & $\begin{array}{l}57 \\
57\end{array}$ & & & & - & & 1 \\
\hline-2 & 0,385 & $1 \varnothing 7 \pi$. & 22,0 & 19,0 & 12,0 & 180 & 84 & 94 & 49 , & 6,8 & 41 . & \\
\hline
\end{tabular}

В элементах серии «П» анкеровка арматуры осуществлялась при помощи утолщеннй на концах арматурных стержней, получаемых высадкой. В серии «O» арматура анкеровалась на забетонированные по торцам металлические плитки $6 \times 6 \mathrm{~cm}$, через которые она пропускалась. 


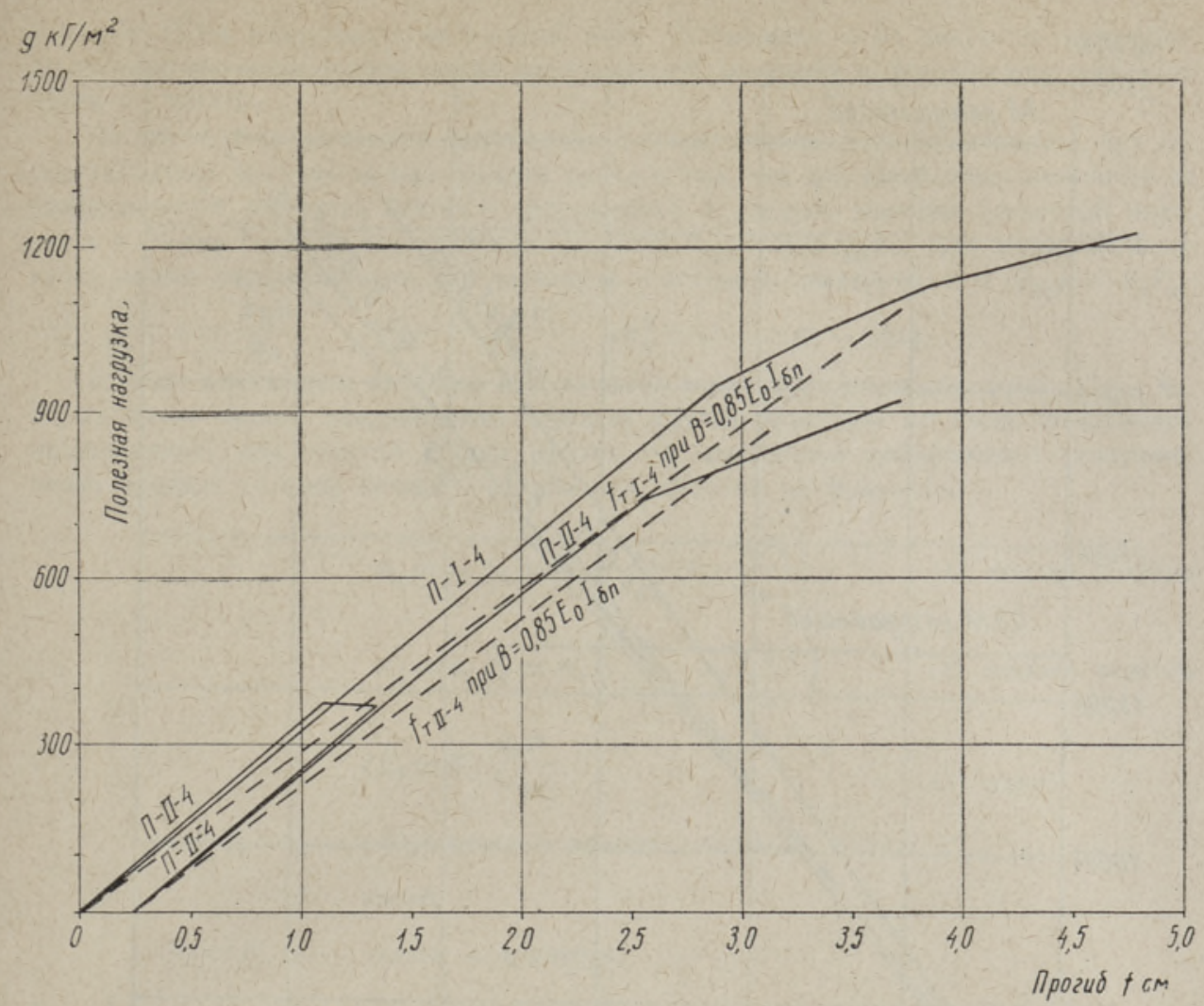

Pнс. 1.

Первоначально арматура натягивалась на формы механическим способом. Однако этот метод имел технические недостатки и был трудоемким. Поэтому в серии « $\mathrm{O}$; впервые было применено электротермическое последующее натяжение арматуры, по крытой расплавляющейся антикоррозионной битумной обмазкой. Сущность этого нового метода заключается в том, что при пропускании электрического тока через арматуру в готовом изделии битумная обмазка размягчается, нарушая сцепление с арматурой, что позволяет последней удлнняться в изделии. После выключения электрического тока арматура анкеруется на торцовых пластинках, а обмазка вновь отвердевает, обеспечивая сцепление арматуры с бетоном.

Таблица 2

\begin{tabular}{|c|c|c|c|c|c|}
\hline № & $\begin{array}{c}M_{\text {pasp }}^{\text {Teop }}, \\
\kappa \Gamma M\end{array}$ & $\xi_{\mathrm{p}}=\frac{x_{\mathrm{p}}}{h_{0}}$ & $\begin{array}{c}M_{\text {разр }}^{\text {фак }} \\
\kappa \Gamma M\end{array}$ & $\frac{M_{\mathrm{p}}^{\text {teop }}}{M_{\mathrm{p}}^{\text {фak }}}$ & $\begin{array}{c}\text { Предвари- } \\
\text { тельное на- } \\
\text { пряжение } \\
\sigma_{0}, \quad \kappa \Gamma / c \mu^{2}\end{array}$ \\
\hline $\begin{array}{l}\Pi-\mathrm{I}-4 \\
\Pi-\mathrm{II}-4 \\
0-1 \\
0-2 \\
0-3\end{array}$ & $\begin{array}{r}1460 \\
2538 \\
307 \\
307 \\
307\end{array}$ & $\begin{array}{l}0,528 \\
0,334 \\
0,154 \\
0,154 \\
0,154\end{array}$ & $\begin{array}{c}1603 \\
2525 \\
307 \\
332,5 \\
330,5\end{array}$ & $\begin{array}{l}1,098 \\
0,995 \\
1,00 \\
1,095 \\
1,075\end{array}$ & $\begin{array}{l}1200 \\
1100 \\
1830 \\
5270 \\
1780\end{array}$ \\
\hline
\end{tabular}

В табл. 2 приведены данные о несущей способности испытанных элементов.

Вычисление значений теоретических разрушающих моментов производилось по формуле

$$
M_{\text {разр }}^{\text {теор }}=R_{\text {и }} b h_{0}^{2} \xi_{\mathrm{p}}\left(1-0,5 \xi_{\mathrm{p}}\right), \quad \text { где } \xi_{\mathrm{p}}=\frac{x_{\mathrm{p}}}{h_{0}}=\frac{F_{\mathrm{н}} \sigma_{\mathrm{\tau}}}{b h_{0} R_{\mathrm{u}}} \text {. }
$$




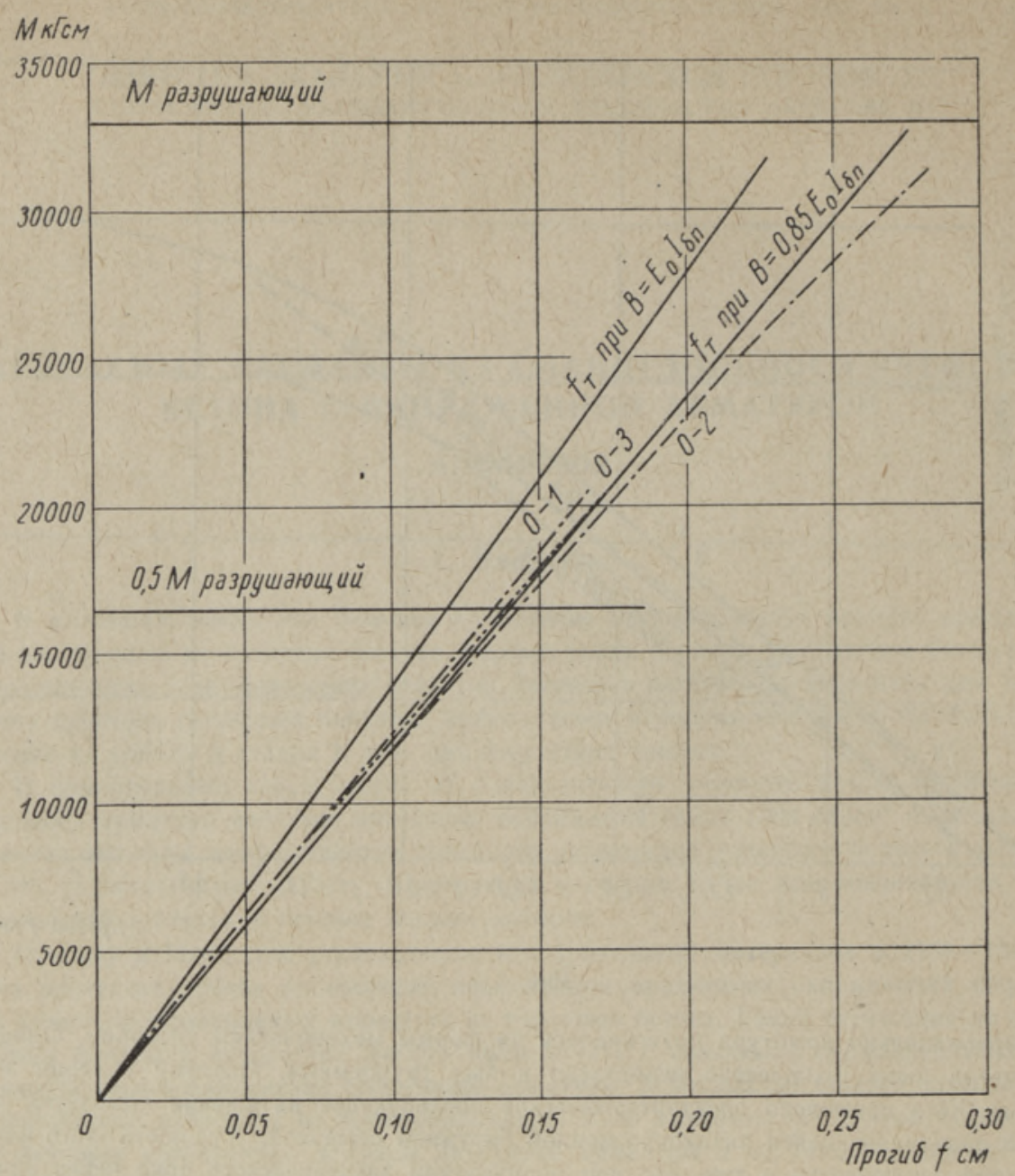

Рнс. 2.

Таблица 3

\begin{tabular}{|c|c|c|c|c|c|c|c|c|c|c|}
\hline & \multicolumn{3}{|c|}{$0-1$} & \multicolumn{3}{|c|}{$0-2$} & \multicolumn{3}{|c|}{$0-3$} \\
\hline & & \multicolumn{9}{|c|}{ по формуле } \\
\hline & & (1) & (2) & (3) & (1) & (2) & (3) & (1) & (2) & (3) \\
\hline Измеренные & $\begin{array}{c}\varepsilon \cdot 10^{-5} \\
f_{\mathrm{CM}}\end{array}$ & $\begin{array}{c}19,6 \\
-\end{array}$ & $\begin{array}{c}6,0 \\
-\end{array}$ & $\frac{-}{0,040}$ & 46,3 & $\begin{array}{c}19,85 \\
-\end{array}$ & 0,129 & $\begin{array}{c}17,0 \\
-\end{array}$ & $\frac{6,65}{-}$ & 0,040 \\
\hline 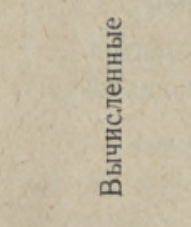 & $\begin{array}{l}\sigma \kappa \Gamma / c \mu^{2} \\
N_{0} \kappa \Gamma \\
N_{0 \mathrm{cp}} \\
\sigma_{0 \mathrm{cp}} \\
N_{0} / N_{0 \mathrm{cp}}\end{array}$ & $\begin{array}{r}8,06 \\
788\end{array}$ & $\begin{array}{c}2,55 \\
677 \\
705 \\
1830 \\
0,96\end{array}$ & $\frac{-}{650}$ & $\begin{array}{r}19,00 \\
1856\end{array}$ & $\begin{array}{l}8,12 \\
2145 \\
2030 \\
5270 \\
1,055\end{array}$ & 2090 & $\begin{array}{l}7,00 \\
684\end{array}$ & $\begin{array}{c}2,73 \\
724 \\
686 \\
1780 \\
1,055\end{array}$ & - \\
\hline
\end{tabular}


Элемент П-І-4 разрушился по сжатой зоне. Остальные - от текучести арматуры. Отношение значений теоретических разрушающих моментов к фактическим составило $99,5 \%-109,8 \%$.

На рис. 1, 2 представлены фактические и теоретические прогибы элементов, при сопостановлении которых можно сделать вывод о том, что для изгибаемых элементов из сланцезольного ячеистого бетона с напряженной арматурой значение расчетной жесткости в стадии I можно определять по формуле $B_{\mathrm{kpI}}=0,85 I_{6 \mathrm{n}} E_{0}$. Для стадий II жесткость можно определять как для элементов с обычной арматурой, т. е. $B_{\mathrm{kp}} \mathrm{II}=E_{a} F_{a}$. $\cdot h_{0}^{2} \cdot \frac{c}{\psi_{a}} ; \alpha=\frac{2 \mu n}{\psi_{a}} \quad \Psi_{a}=\frac{1+\mu n}{2,5} \quad c h_{0}^{2}=(1-x)(1-0,5 x)$.

В табл. 3 приведены данные о напряжениях в арматуре элементов серии «O», в ко торых кроме одного напряженного стержня другой арматуры не было. Вычисления производились при упругой стадии работы по замеренным деформациям (напряжениям) крайних волокон бетона и выгиба при обжатии по формулам

$$
\begin{array}{rlr}
\sigma_{6}=\varepsilon_{6} E_{0}=N_{0}\left(\frac{1}{F_{6 \mathrm{n}}}+\frac{e_{0} y_{6}}{J_{6 \mathrm{n}}}\right) & \text { (1) нижнее волокно } \\
\sigma_{6}^{\prime}=\varepsilon_{6}^{\prime} E_{0}=N_{0}\left(\frac{1}{F_{6 \mathrm{n}}}-\frac{e_{0} \cdot y_{6}^{\prime}}{J_{6 \mathrm{n}}}\right) & \text { (2) верхнее волокно } \\
f E_{0}=N_{0} \frac{e_{0} l^{2}}{8 J_{6 \mathrm{n}}} & \text { (3) выгиб }
\end{array}
$$

где $\sigma_{6}$ и $\sigma_{6}^{\prime}-$ напряжения в крайних волокнах бетона,

$$
f \text { - выгиб, }
$$

$$
N_{0}=F_{\mathrm{H}} \sigma_{0}-\text { усилие в арматуре, }
$$

$F_{6 n}, J_{6 n}, e_{0}, y_{6}$ и $y_{6}^{\prime}$ - геометрические характеристики приведенного сечения.

Как видно из табл. 3 , наибольшее отклонение вычисленных значений $N_{0}$ имеет место в $0-1$, где оно составляет $+11-8 \%$, что говорит о применимости формул (1), (2), (3). Возникновение трещин в растянутой зоне наших опытных элементов наиболее точно можно было определить по формуле $M_{\mathrm{T}}=\left(k R_{\mathrm{p}}+\sigma_{6}\right) W_{6 n}$ (4). Отклонения вычисленных по ней значений моментов трещинообразования от фактических моментов составили $-9,5+5,6 \% . \kappa=\frac{R_{\mathrm{pu}}}{R_{\mathrm{p}}}$ по нашим опытам равно 1,8 . Наблюдения за опытными призмами из сланцезольного яченстого бетона с напряженной арматурой показали, что при $\sigma_{6}=\frac{1}{3} R_{\text {nр }}$ и $\sigma_{0}=2270-2800 \kappa \Gamma / \mathrm{cm}^{2}$ потери напряжения в арматуре за 500 дней составили около $480 \kappa \Gamma / \mathrm{cm}^{2}$, или $17-19 \%$ от первоначального.

\section{Выводы}

Увеличение жесткости изгибаемых элементов из сланцезольного ячеистого бетона можно осуществить, применяя напряженную стержневую арматуру. Наиболее эффективным является применение последующего электротермического натяжения арматуры с временно расплавляющимися битумными обмазками. Для определення напряжений в бетоне конструкций могут быть использованы формулы (1), (2), (3), а для определения значения момента образования трешин формула (4). Потери предварительного напряжения арматуры во времени являются небольшими и допустимыми с точки зрения сохранения предварительных напряжений. 


\section{PINGESTATUD ARMATUURIGA POLLEVKIVITUHK-MULLBETOONIST PAINDEELEMENDID}

\section{A. Morozov}

\section{Resümee}

Artiklis esitatakse pingestatud varrasarmatuuriga põlevkivituhk-mullbetoonist paindeelementide uurimise tulemused. Bituumenvōōbaga kaetud armatuuri järelpingestamiseks soovitatakse elektrotermilist meetodit.

Eesti NSV Teaduste Akadeenia .

Saabus toimetusse Ehituse ja Ehitusmaterjalide Instituut

14. III 1963

\section{OIL SHALE FLY ASH CELLULAR CONCRETE FLEXURAL MEMBERS WITH STRESSED REINFORCEMENT}

\section{A. Morozov}

\section{Summary}

The results of experimental research of oil shale fly ash cellular concrete flexural members with stressed bar-reinforcement are presented in this paper. The author recommends the use of electro-thermic stressing of reinforcement, covered with bituminous coating after the concrete has been cured.

Academy of Sciences of the Estonian S.S.R., Institute of Building and Building Materials

Received March 14th, 1963 